Exploring UK foundation doctors' perceptions surrounding raising concerns in the workplace

Khojasta Talash ${ }^{1,2}$, Lorraine Corfield ${ }^{3,4}$, Natalie Latcham ${ }^{5}$, Claire Lavelle ${ }^{6}$, Richard A Williams ${ }^{7}$, and Laura L Machin ${ }^{2}$.

${ }^{1}$ Manchester University NHS Foundation Trust, Manchester, UK.

${ }^{2}$ Lancaster Medical School, Lancaster University, Lancaster, UK.

${ }^{3}$ University Hospital of North Midlands NHS Trust, Keele, UK.

${ }^{4}$ Keele Medical School, Keele University, Keele, UK.

${ }^{5}$ University Hospitals of Morecambe Bay NHS Foundation Trust, Lancaster, UK.

${ }^{6}$ Wirral GP Specialty Training Scheme, Wirral, UK.

${ }^{7}$ Department of Management Science, Lancaster University, Lancaster, UK.

Corresponding author:

Khojasta Talash

Institution: Manchester University NHS Foundation Trust

Postal address: Manchester University NHS Foundation Trust, Oxford Road, Manchester, UK, M13 9WL

Email: Khojasta.Talash@doctors.org.uk

Telephone: 01524594973 


\section{Exploring UK foundation doctors' perceptions surrounding raising concerns in the workplace}

This study aims to explore the perceptions of foundation doctors (FDs) in the United Kingdom (UK) surrounding raising concerns in the workplace. An online survey was sent to all FDs in the UK between February and March 2018. Respondents were asked what they had observed or experienced that had been ethically challenging during their foundation training. The qualitative responses were coded into themes. Reasons why FDs wished to raise concerns in the workplace included disagreements about clinical decisions within the team, insufficient availability of resources, lack of senior colleague support and having to work beyond their competencies. Challenges faced by FDs when raising concerns included organisational resistance to change, difficulties in communicating ideas to those higher up in the hierarchy and the emotional stress of whistleblowing regarding senior colleagues. Perceived consequences of raising concerns included negative impact on FDs' reputation and career, and fear of bullying. To overcome these barriers, changes within organisations at all levels must take place in order to provide an environment where FDs are encouraged to raise concerns and thus make positive changes to their work environments for themselves, their colleagues and patients.

Keywords: organisations; mentoring; vocational education \& training; learning in the professions; workplace learning 


\section{Introduction}

Organisational factors such as poor staffing, lack of senior support and shortage of beds are considered to be challenging and stressful for junior doctors (McDougall and Sokol 2008; Williams et al. 1997). Initially, junior doctors struggle to report their concerns on quality and safety (Carr et al. 2016), although they are thought to gain confidence about speaking up with increased clinical experience (Beament and Mercer 2016). There have been calls for more support to be provided for junior doctors, as this can impact on their job satisfaction, the quality of care they provide to patients, as well as their own health (Rogers, Creed, and Searle 2014). Despite this, junior doctors sometimes find themselves in situations where there is little or no senior support as evidenced by the UK case of Dr. Bawa-Garba where the lack of senior support was a factor in the junior doctor's error (Cohen 2017).

The culture of organisations can also present challenges for junior doctors when whistleblowing (Francis 2015). The presence of a hierarchy can mean junior members of the team feel unwilling to speak up to senior colleagues (Samuel et al. 2012), and experience resistance to the changes they propose (Goodwin 2019). Leadership training has been proposed to help junior doctors learn how to have their ideas heard (Gilbert et al. 2012), and a curriculum on ethics and law has been proposed to support Foundation Doctors (FDs) for the challenges they face in the first two years since qualifying (Machin et al. 2020). Yet, organisations often regard the junior doctor's role as that of service provision and as they become engrossed in this, their leadership skills and potential become suppressed (Bagnall 2012), and time away from the ward for training becomes harder to justify (Kirkham and Baker 2012).

Junior doctors fear the perceived consequences of whistleblowing, such as isolation from their team (Martinez et al. 2017), bullying (Holt 2015), and negatively impacting on their 
career, as illustrated by the case of the UK whistleblowing junior doctor, Chris Day (Rimmer 2018). It has been suggested that a 'no-blame culture' should be adopted by organisations (Markwell and Wainer 2009), creating an environment that encourages and protects those who speak up (Førde and Aasland 2008; Reiser 1994; Vivekananda-Schmidt and Vernon 2014). Such organisation-wide movements are considered to increase the likelihood of positive change in individuals as they feel part of a bigger change (Solomon et al. 1991), where shared values are embraced, and vehicles to drive those changes exist (Reiser 1994). Examples of such changes are the implementation of Schwartz rounds where healthcare staff from all levels can come together to discuss the emotional aspects of their jobs in a safe environment (Oliver 2018), and the call for widespread introduction of clinical ethics support services (Machin and Wilkinson, forthcoming).

Junior doctors, in particular FDs, demonstrate motivation and enthusiasm for making changes in their workplace in order to improve patient care (Bagnall 2012). Organisation leaders should therefore provide an environment that listens to FDs' ideas for quality improvement (Bethune, Roueche, and Hilman 2011; Gilbert et al. 2012). Yet, FDs are rarely asked how to improve the systems within their workplace (Maisonneuve, Lambert, and Goldacre 2014), leaving some FDs feeling undervalued and unsupported by organisation managers and consultants (Bagnall 2012; Gilbert et al. 2012). Providing a supportive environment for FDs will ensure risks of stress and burnout are minimised, thereby securing sufficient staffing to meet tomorrow's patient demand (Markwell and Wainer 2009). In addition, today's FDs will become tomorrow's clinical leaders and so by providing them with the right preparation and support, they will be able to make a positive influence on the future of the culture within our National Health Service (NHS) (Bagnall 2012).

\section{Purpose}


In this paper we will explore the perceptions of FDs in the United Kingdom (UK) surrounding raising concerns in the workplace, in particular the causes, challenges and consequences of speaking up. Accepting individual responsibility and training as important factors, larger changes on all levels within organisations are needed in order for these causes, challenges and consequences to be properly addressed.

\section{The UK Foundation Programme}

The UK Foundation Programme (UK FP) was introduced in 2005 and encompasses the first two years of clinical practice by newly qualified doctors upon graduation from medical school (Collins 2010). Doctors within the programme are referred to as FDs and are the most junior members of the clinical team. The aim of the training programme is to provide a safe environment for FDs to put the knowledge they gained at medical school into practice and gain exposure to a range of different specialties before choosing their future career paths (Collins 2010; UK FP 2019).

The foundation programme is divided into foundation year one and two and has its own curriculum (UK FP 2016). During the first year, FDs are provisionally licensed to practice with the General Medical Council (GMC), and must show evidence of achieving a set of outcomes by the end of the year in order to be able to become fully registered with the GMC with a licence to practice (Kirkham and Baker 2012). In foundation year two, as they become fully registered and have had more experience of the job, FDs are given more responsibility and can start to make more decisions around patient care (Dean and Duggleby 2013). FD training involves rotation through various placements, usually three per year, where they are predominantly in the hospital environment for example on the surgical or medical wards, and they may also have community attachments (UK FP 2019). As with all doctors in training, 
FDs should have a senior doctor available in their clinical settings to provide them with supervision (Dean and Duggleby 2013).

\section{Materials and methods}

An online survey was sent to all FDs in their first and second years of training across the UK over a six week period between February and March 2018. This timeframe was chosen to provide FDs sufficient time to settle into their new jobs (Byrne et al. 2016). As the questionnaire was promoted with support from the UK Foundation Programme Office and the Institute of Medical Ethics, it was not possible to ascertain how many FDs saw the recruitment advert. However based on the total number of filled posts for FDs during the period that the questionnaire was sent out (UK FP 2017; UK FP 2018), it is estimated that 3\% of all FDs completed the survey. This means that similar to previous studies (Dean and Duggleby 2013) a limitation of our study is the relatively small sample size when considered in light of the number of FDs on the two year Foundation Programme. The potential implications of this small sample size includes selection bias and therefore it is worth noting that the views expressed in this paper may not necessarily reflect the experiences of the FDs who did not participate in the project (Machin et al. 2020). Furthermore, our study focuses solely on the perspectives of FDs, thus it does not include the point of view of senior doctors who play an important function in setting the culture in a workplace.

Participation was voluntary and no incentives were provided for completing the survey. All responses were anonymous and respondents had the option to record answers as 'prefer not to say' where they did not want to share certain demographic data, such as age, gender and stage of foundation training. The survey generated both quantitative and qualitative data. Respondents were asked about the ethical and legal training they received in medical school and would wish to have during foundation training. Answers to these questions were 
recorded in a tick-box format to allow ease of answering. Responses were quantitatively analysed to determine the percentages of respondents having covered a topic in medical school and the percentages of respondents wishing to cover a topic in foundation training. Open-ended text boxes were provided in the survey, which generated qualitative data. Respondents were asked what they had observed or experienced that had been ethically challenging during their foundation training. Respondents were asked not to give any details of a specific case and/or disclose any identifiable information, such as a patient's age, medical condition, hospital or treating clinicians. Those answering the question were reminded to use local governance systems to report any significant concerns. Responses to this question were coded into themes with the gender and stage of training of each respondent included in order to monitor for any patterns between these demographics and the answers recorded. Institutional ethical approval was obtained from the Faculty of Health and Medicine at Lancaster University. Approval for this study was also gathered from Health Education England.

\section{Results}

\section{Explanation of some terms used by respondents in the questionnaire}

To familiarise international readers, we provide a brief overview of several terms used by respondents in our questionnaire. As we collected the responses from a questionnaire and not through face to face interviews, these explanations are based on our clinical experience and interactions with clinicians rather than any definitions provided by the respondents:

Registrars (abbreviated to regs) are doctors who are in specialty training programmes having completed foundation training. 
Consultants are doctors that have completed specialty training and are in charge of the clinical team.

Bed blocking refers to beds that are occupied for a long period by patients who may be medically fit but are unable to be discharged to a safe place.

End of life decisions are the decisions made by the clinical team for patients approaching the end of their life. This may involve palliative care decisions, ceiling of treatment, as well as advanced decisions on whether to perform resuscitation if the need arises.

Form 4 is a consent form that is completed by the clinical team on behalf of a patient when the patient is deemed to lack capacity to make a decision regarding a certain medical procedure or treatment. The clinical team must act in the best interests of the patient.

Deprivation of Liberty Safeguards (DoLS) is a part of the Mental Capacity Act 2005 and is used for patients who are unable to provide consent for their care and are deprived of their liberty. The safeguards assess whether the arrangements put in place by the clinical team are necessary and appropriate for the particular patient.

Detention lifting refers to patients assessed in mental health settings who have been detained under a section of the Mental Health Act 2007, for example for assessment and/or treatment of a mental disorder.

\section{Demographics}

In total, 479 FDs across year 1 and year 2 completed the survey. Demographic data are summarised in Table 1. The majority of respondents were female and aged between 25-30 years. Slightly more than half of the respondents were in their second year of training. 
[Insert Table 1 here]

In response to the free text question on the ethical challenges experienced during their training, 229 FDs provided an answer. These answers fell broadly into two areas: concerns about clinical decisions made by the healthcare team, and concerns about poor working environments. We have separated these as whilst both concerns could be addressed by liaising with the individuals concerned and ameliorated by discussion with the team, and therefore be of educational benefit to the FDs, it is more likely that the second concern needs addressing at a higher level.

\section{Clinical decisions}

Two specific areas that were highlighted regarding disagreements with the clinical team were discussions around end of life (EoL) decisions and do not attempt resuscitation (DNAR) status. Seventy-seven percent of respondents reported receiving training on EoL at medical school and $47 \%$ of respondents wanted EoL training as part of their foundation training. Respondents described feeling uncomfortable about certain EoL decisions made by the team or senior colleagues, where the FDs felt the patients were being overtreated as the following quotes from FDs in their first and second year of training illustrate:

'Continuing surgical treatment when a patient with severe learning difficulties with terminal metastatic cervical cancer required multiple procedures. Palliative care was not brought up and consent was obtained without a form 4 despite the patient's inability to consent.' [Female year 1]. 
'Have occasionally disagreed with EoL indecision - patients clearly at end of life but some medical staff not empowered to take action to keep comfortable etc and continue aggressive treatment.' [Female year 1].

'(EoL) decisions, particularly when seniors have continued to investigate or treat even when patients are very frail or clearly dying...' [Female year 2].

Similar challenges were identified with DNAR discussions. Seventy-seven percent of respondents reported receiving training on DNAR decisions at medical school and 57\% of respondents wanted DNAR to be taught further during their foundation training. The following FDs in year 2 who had had DNAR teaching at medical school and stated they would like further training during their foundation years described situations where they felt DNAR decisions were not properly managed:

'Senior doctors feeling that [DNAR] only needs to be put in place when patients are poorly, not prior to that as an advance decision.' [Female year 2].

'Questionable decision making from seniors surrounding [DNAR] decision making and escalation of care.' [Male year 2].

Respondents also identified certain approaches their senior colleagues adopted as concerning, as the following quote from a FD who wanted training on whistleblowing during the foundation programme illustrates:

'Situations where consultant may not seem to realise significance of ethical/legal issue.' [Female year 2].

One respondent also described feeling uneasy about the general attitude of some senior colleagues: 
'Character ethics of registrars apathy towards dealing with a patient, reluctance to take responsibility for their care and assist in registrar level jobs ie reg to registrar referrals across hospitals.' [Female year 1].

One respondent who wanted training on whistleblowing both at medical school and during foundation programme went further to comment on the challenge of trying to express an opinion to someone more senior in the hierarchy:

'Sometimes it is difficult to communicate with senior staff (as the most junior member of the team) with regards to family issues/[DNAR] decisions.' [Female year $1]$.

Another respondent in the second year of foundation training highlighted the benefit of having the opportunity to discuss ethically challenging cases with more senior colleagues:

'I think that having the chance to discuss difficult ethical situations with senior colleagues as they arise is key to gaining experience and confidence.' [Female year 2].

\section{Work environment}

The following two FDs who both agreed they wanted training on being honest and open as foundation doctors described situations when they were asked to perform tasks for which they lacked training or sufficient experience to undertake:

'...Pressure to perform things outside of foundation competencies.' [Male year 2].

'Being requested to do something by a senior colleague that, on reflection, I wasn't comfortable doing.' [Female year 1]. 
Similar issues were described by other respondents such as the following two FDs who both wished to have training on DoLS and assessing mental capacity as part of their foundation training:

‘...Being asked to assess patients for detention or lifting of the detention which I obviously am not qualified to do.' [Male year 1].

'Asked to put DOLs in place and do mental capacity assessments when you have had no formal training on trust procedures, and people often want it doing quickly...' [Female year 2]

In addition, FDs described feeling pressure from bed management to discharge patients due to bed shortages. Again this illustrates the role the organisation plays in creating a challenge for FDs when they felt obligated to speak up, as the below comments from FDs who wished for training on resource allocation demonstrate:

'Feeling pressurised to discharge patients from bed management. Bed blocking due to lack of social care/funding.' [Male year 1].

'Difficulties with pressure from management to discharge patients due to bed crisis.' [Female year 1].

Respondents also described trying to give their best care to patients whilst facing the challenges posed by poor staffing. A cause for raising concerns was that FDs were frequently asked to cover for multiple colleagues at once, as highlighted by the following quotes from FDs who wanted training on whistleblowing:

'Being so under staffed that you feel you can't always perform to the best of your ability.' [Female year 2]. 
'..Shifts not being covered - at all grades from FYs to Consultant and this not being reported.' [Male year 2].

Respondents also described a lack of availability of support from senior colleagues, particularly when making decisions about palliative care and performing examinations as the following quotes from second year FDs illustrate:

'End of life care without availability of sufficient senior support.' [Male F2]

'Performing examinations that I have felt out of my comfort zone with, and not had senior input.' [Female year 2].

Some respondents implied that the staffing problem was accepted by those in the workplace to explain why nothing had been done to resolve it, either from management or those doing the shifts, as the following quote from a doctor who wished for training on whistleblowing highlighted:

'Constantly covering for two or three (at one time FIVE) of my colleagues in an environment that tells me "other people do it, you should too" and gives me no support to fight the poor conditions we suffer.' [Female year 2].

This appeared to create a challenge for those who wished to blow the whistle. In addition, working in such environments makes some doctors feel undervalued as the above FD felt doctors were "constantly taken advantage of and made to comply with a system that is flawed and simply not safe.' [Female year 2].

One respondent in his first year of foundation training described a concerning struggle they face due to the high workload, with the result being lack of sufficient rest for the FD: 
'Making the choice (almost every day) between keeping patients safe and getting appropriate rest break.' [Male year 1].

For some respondents, the perceived consequences to themselves from whistleblowing about the work environment however meant they were reluctant to speak out as the quote below from a doctor who wanted further training on whistleblowing demonstrates:

'Reporting patient safety issues or unsafe working schedules/environment can be met with superficially pleasant response, but ends up with you having a bad reputation within the department.' [Female year 1].

\section{Whistleblowing}

When asked about whistleblowing, $42 \%$ of respondents reported having received training on this at medical school. Fifty percent of respondents stated they would like teaching on whistleblowing in foundation training: this included 92 of those who had had training on whistleblowing at medical school (thus $46 \%$ of those who had training as undergraduates wanted more training during foundation training).

Some respondents specifically mentioned whistleblowing or raising concerns as an ethical challenge they had faced in their foundation year(s). Both of the following doctors who wished for more training on whistleblowing gave examples of times they felt compelled to speak up in the workplace:

'I have had an episode of whistleblowing this year in FY2 were I had to voice my concerns and it was quite stressful for me as this person was my senior colleague.' [Male year 2]. 
'Discussions regarding poor quality of colleagues work - escalating above them and the professional relationships. Whistleblowing and our workplace right...' [Female year 2].

This response also highlights the fear FDs described surrounding the perceived consequences as a result of whistleblowing. These fears were echoed by other respondents such as a female in the second year of her foundation training who did not have any training on whistleblowing at medical school:

'Raising issues in a confidential but professional manner - without repercussions for the whistle blowers.' [Female year 2].

Another doctor, who did have training on whistleblowing, found this particularly challenging when the concern related to senior colleagues:

'I have seen a lot of senior members act in ways I feel is ethically incorrect however the nature of the medical system is that senior members are never questioned or challenged and if they are this usually backfires on the person questioning leading to them being penalised. This causes a fear of standing up for patients ethical rights in fear or bullying in the workplace, gaining a negative reputation and fear of dismissal. The problem lies with the senior staff abusing their power rather which leads to junior staff feeling limited in what they can achieve in terms of ethical and human rights.' [Female year 1].

This response also identifies the perceived consequence of whistleblowing on the FD's career.

\section{Discussion}


In this paper we have explored FDs' perceptions of the causes, challenges and consequences of raising concerns in the workplace. The key issues identified regarding the causes for raising concerns in our study relate to the role of senior colleagues and the work environment.

\section{Causes}

Our results show that FDs sometimes disagree on decisions made by the healthcare team or senior doctors regarding EoL and DNAR, where the FDs feel patients may be overtreated, a finding that concurs with existing literature (Linklater 2010). In relation to the work environment, FDs reported facing situations where they did not have adequate senior support, were asked to work beyond their competencies, and faced pressure from lack of staffing and bed shortages. Previous studies have also identified these organisational factors as causing challenges for FDs (Linklater 2010; McDougall and Sokol 2008; Williams et al. 1997).

\section{Challenges}

In this study, FDs reported facing challenges from senior colleagues and the work environment when raising concerns in the workplace. These consisted of the emotional stress of having to raise concerns about a senior colleague, as well as organisational factors including trying to communicate ideas to those more senior in the hierarchy and facing resistance and reluctance from seniors and managers to making changes in the work environment. These results support previous findings (Bagnall 2012; Francis 2015; Goodwin 2019; Samuel et al. 2012).

\section{Consequences}

Our results have also identified the consequences perceived by FDs of raising concerns in the workplace. FDs reported fear of bullying and gaining a negative reputation within a department and fear that there would be negative impacts on their careers if they were to blow the whistle, findings which are consistent with existing literature (Holt 2015; Martinez 
et al. 2017). It is likely that these fears have been exacerbated in recent years by high profile cases involving junior doctors (Cohen 2017; Rimmer 2018).

\section{What changes can we make?}

A previous study has shown that some junior doctors tend to voice their concerns informally as opposed to reporting them on incident report forms (Hooper et al. 2015), however our study has found that FDs find it difficult to raise their concerns verbally. Whilst this may be due to a lack of experience and over the years they may become more confident about speaking up as they go through their clinical training (Beament and Mercer 2016), FDs are an important group whose views can help us improve systems within our hospitals (Gilbert et al. 2012). As the FDs rotate through wards and departments, they act as the 'eyes and ears of the service' (Department of Health 2015), and organisational leaders should encourage them to share their ideas for quality improvement in order to make positive changes in the workplace (Bethune, Roueche, and Hilman 2011; Francis 2015). This will ensure we have a safe healthcare system for patients (Department of Health 2015; Francis 2015), and will help nurture our FDs into becoming successful future clinical leaders of the NHS (Bagnall 2012). Senior doctors have an important educational and supportive role in training FDs. We have shown that at times FDs question the decision taken by the healthcare team. It is only by an open and educational culture from senior doctors that the FDs will appreciate the nuances that they had perhaps missed and understand the reasons for the senior decision. Equally, a senior doctor who encourages and permits such discussions around complex decisions also has the opportunity to learn through questions raised by FDs.

Our study also suggests that FDs would benefit from ethics teaching during their Foundation training, a finding that concurs with existing literature (Chamsi-Pasha, Chamsi-Pasha, and Albar 2016). However, simply providing further teaching is insufficient in tackling the challenges identified in our study. On the contrary, we have previously identified that FDs' 
confidence for dealing with ethical issues in the workplace was unrelated to their ethics teaching received at medical school (Corfield et al 2020). Furthermore, it has been argued that teaching about whistleblowing without providing the supportive work environment for it to occur in can negatively impact FDs by causing moral distress (Martinez et al. 2017). Instead, organisations should encourage and protect whistleblowers (Førde and Aasland 2008; Reiser 1994; Vivekananda-Schmidt and Vernon 2014), adopt a 'no-blame culture' (Markwell and Wainer 2009), and provide an environment where FDs feel valued by senior members of the hierarchy and able to voice their opinion. As advocated by the Point of Care Foundation, managers should support their teams by openly talking about the work pressures they face and how they can be overcome (Oliver 2018).

\section{Our recommendations}

With this in mind, we propose a set of recommendations for each of the parties involved in the training of FD. We believe educators should ensure FDs are provided with ethics training opportunities to enable them to find effective ways to deal with issues that arise in their workplaces. Senior doctors, especially supervisors, should take on an active teaching role in these training opportunities where possible and engage with FDs in the workplace so that they both feel supported in their clinical work and also able to raise concerns. This should provide FDs with more learning opportunities to better understand the clinical decisions made whilst also providing senior doctors with the opportunity to learn from the issues raised by FDs. In order for these proposed recommendations to have a long lasting impact changes should be implemented at all levels, in particular organisation leaders. Speciality directors, clinical leads and ward managers should encourage open discussions at all levels of the organisation and protect those who raise concerns in the workplace. Finally FDs should consult their educators where they feel there is a gap in their ethics training. With the successful implementation of these proposed changes, we should have a work environment 
where FDs feel able to voice their questions or concerns, without fear of the consequences, and be actively listened to so that positive changes are made to their work environment in response to their concerns, where applicable.

Abandoning the emotive and highly charged term 'whistleblowing' may also go some way towards this, as might more senior members of the organisation taking responsibility for the concern once raised, much as transport officials in the UK do - all one has to do is 'see it' and 'say it' and they will 'sort it'. Without addressing the challenges faced by FDs when raising concerns, we are at risk of allowing moral distress to propagate within our Trusts, which can lead to burn out and demotivation amongst staff resulting in loss of the next generation of our workforce from the NHS. Changes should focus on large organisational movements that will encourage individuals at all levels to implement the improvements into their day-to-day practice.

\section{Future directions}

In depth qualitative research is much needed in order to explore the views of FDs on how to best tackle these causes, challenges and consequences of raising concerns. In addition, the perspectives of educators, senior doctors and organisational leaders should be explored in order to gain a holistic picture on the matter of raising concerns within the NHS workplace. Conducting future research with educators, senior doctors and organisational leaders would also enable researchers to ascertain how best we can implement the proposed changes to allow FDs to be able to raise concerns without fear of any consequences. 


\section{Acknowledgements}

We wish to express our sincere gratitude to the Institute of Medical Ethics (UK) who provided funding for the online survey tool. We are also grateful to the time and support from colleagues within the UK FPO, GMC, HEE and BMA who offered feedback and guidance on the study design, content of the survey, and recruitment. However, the views and opinions expressed in this paper are the authors' own, and do not necessarily reflect the views of the people we acknowledge here.

\section{Declaration of Interest}

None declared by any of the authors. 


\section{References}

Bagnall P. 2012. "Facilitators and Barriers to Leadership and Quality Improvement. The King's Fund Junior Doctor Project." London: The Kings Fund.

https://www.kingsfund.org.uk/sites/default/files/facilitators-barriers-leadership-qualityimprovement-pippa-bagnall-leadership-review2012-paper.pdf

Beament T, Mercer SJ. 2016. "Speak up! Barriers to Challenging Erroneous Decisions of Seniors in Anaesthesia." Anaesthesia 71:1332-40. doi:10.1111/anae.13546

Bethune R, Roueche A, Hilman T. 2011. "Is Quality of Care Improving? Improvement Efforts Need to be Targeted at Junior Doctors.” British Medical Journal 342:d1323. doi: $\underline{10.1136 / \mathrm{bmj} . \mathrm{d} 1323}$

Byrne D, Buttrey S, Carberry C, Lydon S, O’Connor P. 2016. "Is there a Risk Profile for the Vulnerable Junior Doctor?” Irish Journal of Medical Science 185:603-9. doi: $\underline{10.1007 / \mathrm{s} 11845-015-1316-3}$

Carr S, Mukherjee T, Montgomery A, Durbridge M, Tarrant C. 2016. "Developing the 'Gripes' Tool for Junior Doctors to Report Concerns: a Pilot Study.” Pilot and Feasibility Studies 2:60. doi:10.1186/s40814-016-0100-0

Chamsi-Pasha H, Chamsi-Pasha M, Albar MA. 2016. "Pragmatic Message to Junior Doctors." Postgraduate Medical Journal 92:418-20. doi:10.1136/postgradmedj-2015-133855 
Cohen D. 2017. "Back to Blame: the Bawa-Garba Case and the Patient Safety Agenda." British Medical Journal 359:j5534. doi:10.1136/bmj.j5534

Collins J. 2010. "Foundation for Excellence: An Evaluation of The Foundation Programme." London: Medical Education England.

https://www.copmed.org.uk/images/docs/1314194412 fhWH foundation for_excellence an evaluation of the fou.pdf

Corfield L, Williams RA, Lavelle C, Latcham N, Talash K, Machin L. 2020. "Prepared for practice? UK Foundation doctors' confidence in dealing with ethical issues in the workplace". Journal of Medical Ethics doi: http://dx.doi.org/10.1136/medethics-2019-105961

Dean BJ, Duggleby PM. 2013. 'Foundation Doctors' Experience of their Training: A Questionnaire Study.” Journal of the Royal Society of Medicine short reports 4:1-7. doi: $\underline{10.1258 / \text { shorts.2012.012095 }}$

Department of Health. 2015. "Culture Change in the NHS: Applying the Lessons of the Francis Inquiries. "

https://assets.publishing.service.gov.uk/government/uploads/system/uploads/attachment data /file/403010/culture-change-nhs.pdf

Førde R, Aasland OG. 2008. "Moral Distress Among Norwegian Doctors.” Journal of Medical Ethics 34:521-5. doi:10.1136/jme.2007.021246 
Francis RA. 2015. "Freedom to Speak Up: An Independent Review Into Creating an Open and Honest Reporting Culture in the NHS: Report." http://freedomtospeakup.org.uk/wpcontent/uploads/2014/07/F2SU_web.pdf

Gilbert A, Hockey P, Vaithianathan R, Curzen N, Lees P. 2012. "Perceptions of Junior Doctors in the NHS about their Training: Results of a Regional Questionnaire.” British Medical Journal Quality Safety 21:234-8. doi:10.1136/bmjqs-2011-000611

Goodwin D. 2019. "NHS Inquiries and the Problem of Culture." The Political Quarterly 90:202-9. doi: $\underline{10.1111 / 1467-923 X .12693}$

Holt K. 2015. "Whistleblowing in the NHS.” British Medical Journal 350:2300. doi: $\underline{10.1136 / \mathrm{bmj} . \mathrm{h} 2300}$

Hooper P, Kocman D, Carr S, Tarrant C. 2015. “Junior Doctors' Views on Reporting Concerns about Patient Safety: a Qualitative Study.” Postgraduate Medical Journal 91:2516. doi:10.1136/postgradmedj-2014-133045

Kirkham D, Baker P. 2012. “Twelve Tips for Running Teaching Programmes for Newly Qualified Doctors.” Medical Teacher 34:625-630. doi: $\underline{\text { 10.3109/0142159X.2012.668243 }}$

Linklater GT. 2010. "Educational Needs of Foundation Doctors Caring for Dying Patients." The Journal of the Royal College of Physicians of Edinburgh 40:13-8.

doi: $\underline{10.4997 / J R C P E .2010 .104}$ 
Machin LL, Latcham N, Lavelle C, Williams RA, Corfield L. 2020. "Exploring the Perceived Medical Ethics and Law Training Needs of UK Foundation Doctors.” Medical Teacher 42:92-100 doi:10.1080/0142159X.2019.1665636

Machin LL, Wilkinson M. Forthcoming. "Making the (Business) Case for Clinical Ethics Support in the UK."

Maisonneuve JJ, Lambert TW, Goldacre MJ. 2014. “Doctors' Views about Training and Future Careers expressed one Year after Graduation by UK-Trained Doctors: Questionnaire Surveys Undertaken in 2009 and 2010.” BMC Medical Education 14:270. doi: $\underline{10.1186 / \mathrm{s} 12909-014-0270-5}$

Markwell AL, Wainer Z. 2009. "The Health and Wellbeing of Junior Doctors: Insights from a National Survey." The Medical Journal of Australia 191:441-4. doi:https://doi.org/10.5694/j.1326-5377.2009.tb02880.x

Martinez W, Lehmann LS, Thomas EJ, Etchegaray JM, Shelburne JT, Hickson GB, Brady DW, et al. 2017. "Speaking up about Traditional and Professionalism-Related Patient Safety Threats: a National Survey of Interns and Residents." British Medical Journal Quality Safety 26:869-80. doi:10.1136/bmjqs-2016-006284

McDougall R, Sokol DK. 2008. "The Ethical Junior: a Typology of Ethical Problems Faced by House Officers." Journal of the Royal Society of Medicine 101:67-70. doi: $\underline{10.1258 / \mathrm{jrsm} .2007 .070412}$ 
Oliver D. 2018. "David Oliver: Moral distress in Hospital Doctors.” British Medical Journal 360:k1333. doi:10.1136/bmj.k1333

Reiser SJ. 1994. "The Ethical Life of Health Care Organizations.” The Hastings Center Report 24:28-35. doi: $10.2307 / 3563464$

Rimmer A. 2018. "Junior Doctor Whistleblowers Need Protection they can Trust, says Robert Francis.” British Medical Journal 361:k1883. doi:10.1136/bmj.k1883

Rogers ME, Creed P, Searle J. 2014. “Emotional Labour, Training Stress, Burnout, and Depressive Symptoms in Junior Doctors." Journal of Vocational Education \& Training 66:232-48. doi: $10.1080 / 13636820.2014 .884155$

Samuel R, Shuen A, Dendle C, Kotsanas D, Scott C, Stuart RL. 2012. "Hierarchy and Hand Hygiene: would Medical Students Speak up to Prevent Hospital-Acquired Infection?” Infection Control \& Hospital Epidemiology 33:861-3. doi:10.1086/666634

Solomon MZ, Jennings B, Guilfoy V, Jackson R, O’Donnell L, Wolf SM, Nolan K, KochWeser D, Donnelly S. 1991. "Toward an Expanded Vision of Clinical Ethics Education: from the Individual to the Institution.” Kennedy Institute of Ethics Journal 1:225-45. doi: $\underline{10.1353 / \text { ken. } 0.0051}$ 
UK Foundation Programme. 2016. "The Foundation Programme Curriculum 2016." https://foundationprogramme.nhs.uk/wpcontent/uploads/sites/2/2019/11/FP_Curriculum_2016_V2-1_0.pdf

UK Foundation Programme. 2017. "Recruitment Stats and Facts Interim Report April 2017." https://www.foundationprogramme.nhs.uk/wp-content/uploads/sites/2/2019/11/Stats-andFacts-FP-2017 0.pdf

UK Foundation Programme. 2018. "F2 Career Destinations Report 2018." https://www.foundationprogramme.nhs.uk/wp-content/uploads/sites/2/2019/11/F2-CareerDestinations-Report_FINAL-2018.pdf

UK Foundation Programme. 2019. "UK Foundation Programme Rough Guide.” https://www.foundationprogramme.nhs.uk/wp-content/uploads/sites/2/2019/12/UKFP$\underline{\text { Rough-Guide-2019-V2.pdf }}$

Vivekananda-Schmidt P, Vernon B. 2014. "FY1 Doctors' Ethicolegal Challenges in their First Year of Clinical Practice: an Interview Study." Journal of Medical Ethics 40:277-81. doi: $\underline{10.1136 / \text { medethics-2011-100391 }}$

Williams S, Dale J, Glucksman E, Wellesley A. 1997. "Senior House Officers' Work Related Stressors, Psychological Distress, and Confidence in Performing Clinical Tasks in Accident and Emergency: a Questionnaire Study.” British Medical Journal 314:713-18. doi: $10.1136 / \mathrm{bmj} .314 .7082 .713$ 

Table 1. Demographic data of respondents

\begin{tabular}{|c|c|c|c|}
\hline Parameter & Values & $\begin{array}{l}\text { Number of } \\
\text { respondents }\end{array}$ & $\begin{array}{l}\text { Percentage of respondents (to the } \\
\text { nearest whole number) }\end{array}$ \\
\hline \multirow[t]{3}{*}{ Gender } & Female & 308 & $64 \%$ \\
\hline & Male & 158 & $33 \%$ \\
\hline & Prefer not to say & 13 & $3 \%$ \\
\hline \multirow[t]{5}{*}{ Age } & $20-24$ & 136 & $28 \%$ \\
\hline & $25-30$ & 292 & $61 \%$ \\
\hline & $30-34$ & 30 & $6 \%$ \\
\hline & $35+$ & 16 & $3 \%$ \\
\hline & Prefer not to say & 5 & $1 \%$ \\
\hline \multirow{3}{*}{$\begin{array}{l}\text { Stage of } \\
\text { training }\end{array}$} & Year 1 & 218 & $46 \%$ \\
\hline & Year 2 & 254 & $53 \%$ \\
\hline & Prefer not to say & 7 & $1 \%$ \\
\hline
\end{tabular}


Table 1. Demographic data of respondents 